\title{
Absorption Spectroscopy of an Individual Fano Cluster
}

\author{
Mustafa Yorulmaz ${ }^{\dagger \neq}$, Anneli Hoggard ${ }^{\dagger \neq}$, Hangqi Zhao ${ }^{\ddagger \|}$, Fangfang Wen $^{\dagger \neq}$,

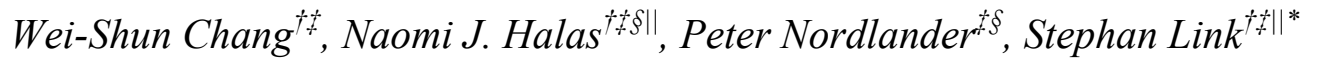

${ }^{\dagger}$ Department of Chemistry, ${ }^{\S}$ Department of Physics and Astronomy, "Department of Electrical and Computer Engineering, 'Laboratory for Nanophotonics, Rice University, 6100 Main Street, Houston, Texas 77005, United States

\section{KEYWORDS}

Surface plasmons, Fano resonance, near-field coupling, single-particle spectroscopy, photothermal imaging 


\section{ABSTRACT}

Plasmonic clusters can exhibit Fano resonances with unique and tunable asymmetric lineshapes, which arise due to the coupling of bright and dark plasmon modes within each multi-particle structure. These structures are capable of generating remarkably large local electromagnetic field enhancements, and should give rise to high hot carrier yields relative to other plasmonic nanostructures. While the scattering properties of individual plasmonic Fano resonances have been characterized extensively both experimentally and theoretically, their absorption properties, critical for hot carrier generation, have not yet been measured. Here, we utilize single particle absorption spectroscopy based on photothermal imaging to distinguish between the radiative and non-radiative properties of an individual Fano cluster. In observing the absorption spectrum of individual Fano clusters, we directly verify the theoretical prediction that while Fano interference may be prominent in scattering, it is completely absent in absorption. Our results provide microscopic insight into the nature of Fano interference in systems of coupled plasmonic nanoparticles, and should pave the way for the optimization of hot carrier production using plasmonic Fano clusters. 


\section{INTRODUCTION}

Collective oscillations of the conduction band electrons in metal nanostructures, known as surface plasmons, exhibit unique optical properties sensitive to their size and shape and the refractive index of the surrounding medium. ${ }^{1}$ These highly tunable surface plasmon resonances have enabled a wide range of diverse applications ranging from nanoscale sensing, ${ }^{2,3}$ enhanced spectroscopies $^{4-6}$ to vivid color generation. ${ }^{7}$ Carefully designed nanoparticle assemblies provide increased control over the spectral lineshape. ${ }^{8-11}$ Similar to atoms in a molecule, plasmonic clusters exhibit hybridized modes that arise from near-field coupling. ${ }^{12-15}$ The lineshape of these plasmon modes can be controlled by tuning the coupling strength, which largely depends on the distance and relative orientation of the individual nanoparticles in a cluster. ${ }^{16-19}$

Further control of light scattering can be achieved through mode interference, which is determined by the relative phase of the different plasmon resonances that can be excited in a nanostructure. $^{20,21}$ This effect is due to the fact that light scattering from a nanostructure is nonlocal, i.e. proportional to the square of the total dipole moment. Thus, when several dipolar modes can be excited, constructive and destructive interference can occur. If all relevant dipole moments are oscillating in phase, a strongly broadened superradiant mode is formed. If the individual dipole moments oscillate out of phase, the total dipole moment can vanish and the mode is subradiant. The radiance of a plasmon mode is determined by hybridization of the plasmon resonances associated with the individual structural parts of the nanostructure and can be controlled by structural symmetry breaking or radiative coupling. A particularly interesting situation is weak coupling when superradiant and subradiant modes interfere, resulting in a strongly asymmetric lineshape in the scattering spectrum ${ }^{22}$ known as a Fano resonance. ${ }^{23}$ In this case, incident light couples efficiently to the broad superradiant mode that transfers some of its 
energy through dispersive near-field coupling to the narrow, optically dark subradiant mode. ${ }^{18,20}$ The spectrum of a Fano resonance is composed of a broad, intense scattering feature centered around the superradiant mode with a narrow dip between the sub- and superradiant modes. At wavelengths corresponding to this "Fano dip", far-field radiation is minimized. ${ }^{18,20,21}$ Fano resonances have been observed in numerous plasmonic systems, such as metamaterials, ${ }^{24-26}$ ringdisk cavities, ${ }^{27,28}$ and coupled clusters of nanoparticles. ${ }^{8,} 13,17,18,20,29-41$ Unlike atomic systems where Fano resonances were first discovered in absorption spectra, for plasmonic systems Fano interferences cannot be observed in absorption because in contrast to scattering, absorption is local and can be evaluated as an integral of the local dissipation at each point of the structure. ${ }^{21}$

Due to the sensitivity to changes in their dielectric environment, their narrow widths, and their strongly enhanced near-field properties, Fano resonant nanostructures have become important for sensing applications. ${ }^{21,42-44}$ A particularly high figure of merit of $1000 \mathrm{~nm}$ per refractive index unit was achieved using heptamers, i.e. nanoparticle clusters consisting of a central disk surrounded by six disks. ${ }^{20,45}$ The Fano resonance can be tuned to desired wavelength ranges by varying the metal, nanoparticle sizes, interparticle distances, as well as overall geometric arrangement. ${ }^{14,20,28,40,46,47}$ Recent studies showed how arrays of nanostructures supporting Fano resonances in the near infrared region can be used to determine the conformation and thickness of adsorbed protein monolayers. ${ }^{46}$

The ability of Fano resonances to enhance hot carrier generation has also attracted significant attention in light harvesting applications. ${ }^{48,49}$ Hot carriers are generated from the non-radiative decay of surface plasmons in a manner similar to Landau damping in extended structures. ${ }^{50} \mathrm{An}$ increase in the efficiency of photovoltaic devices was demonstrated using plasmonic Fano resonant clusters by photo-induced doping of graphene. ${ }^{49}$ Even without direct characterization of 
the absorption of these nanostructures, spectral analysis shows that the carrier generation efficiency is directly related to the absorption of the metal antenna. ${ }^{49}$ Similar results of enhanced absorption close to the Fano resonance in a semiconductor nanostructure were also deduced from photocurrent measurements. ${ }^{51}$ The development of a method for direct characterization of the wavelength-specific absorption properties of these complex nanostructures is therefore important for the design and optimization of light harvesting platforms for energy generation and catalysis. $^{52,53}$

While classical electromagnetic modeling can accurately simulate the optical properties of plasmonic nanostructures, ${ }^{54,55}$ independent experimental characterization provides indispensable feedback, especially as nanostructures become increasingly complex. ${ }^{56}$ While absorption and scattering show close resemblance for small, simple nanoparticles $(<50 \mathrm{~nm})$, they can be substantially different for larger nanostructures. Single-particle dark-field microspectroscopy has been used extensively to characterize the far-field properties of plasmonic Fano resonances, but does not directly provide wavelength-dependent absorption information. With important emerging applications such as photodetectors ${ }^{49}$ and photocatalysts ${ }^{57}$ whose properties are more directly related to light absorption by the nanostructure, it is clearly important to develop experimental methods to separately measure absorption and scattering distinctly at the individual nanostructure level.

In this paper, we study the absorption spectrum of a single plasmonic nanoparticle oligomer- a decamer consisting of a central disk surrounded by nine smaller disks- that supports a strong Fano resonance (Fano cluster), and its individual components. By comparing its absorption spectrum with its scattering spectrum we are able to separately characterize both the nonradiative and radiative properties of this plasmonic Fano cluster as well as its components. We 
show that the asymmetric lineshape characteristic of a Fano resonance is noticeably absent in the absorption spectrum, which is instead a simple symmetric resonance centered at the subradiant mode. Establishing the distinct absorption properties of a plasmonic structure through direct experimental means supports the development of plasmonic nanostructures for optimizing their absorption-related properties, such as hot carrier generation, ${ }^{58,59}$ that could complement their already promising sensing and surface-enhanced spectroscopy applications.

\section{RESULTS AND DISCUSSION}

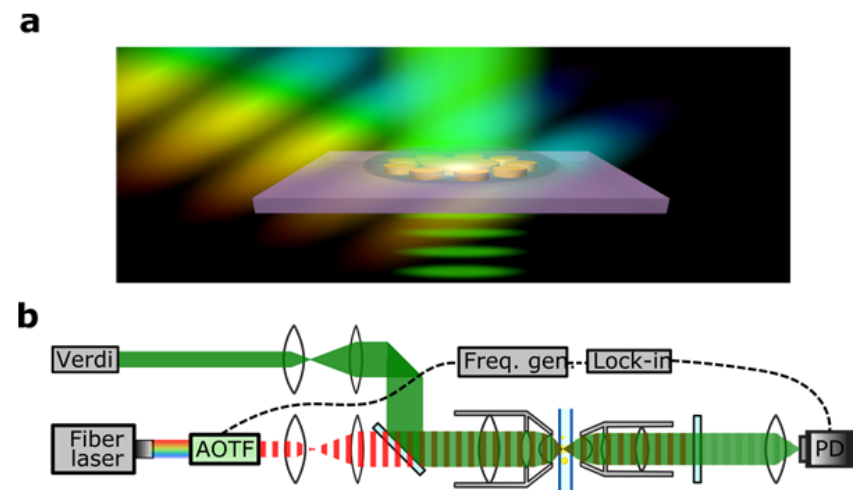

Figure 1. a) Schematic illustration of the photothermal spectroscopy technique. Wavelength tunable excitation from a white light laser is modulated and heats individual nanostructures and their surrounding due to plamson absorption. Transmission changes of a green probe beam are modulated and detected via lock-in amplification. $b$ ) Description of the experimental setup (see text for details). AOTF: acousto optical tunable filter; PD: photodiode; Freq. Gen.: frequency generator; Lock-in: lock-in amplifier.

We perform wavelength-dependent photothermal measurements in a transmission geometry for single particle absorption spectroscopy, illustrated Fig. 1. Excitation wavelengths with 2-6 nm bandwidth are selected from a broadband pulsed laser source (Fianium Whitelaser SC450) using an acousto-optical tunable filter (AOTF). To extend the spectral range of our absorption spectrometer compared to our previous work $^{60}$, we used two different AOTFs covering two wavelength ranges, i.e. VIS (400 - $750 \mathrm{~nm})$ and NIR $(650-1100 \mathrm{~nm})$ shown in Figs. S1a and $\mathrm{S} 1 \mathrm{~b}$, respectively. The laser beam intensities were modulated by the AOTFs to create a time- 
dependent thermal lens around an individual nanostructure due to the temperature-induced refractive index changes of the surrounding medium following plasmon absorption and nonradiative relaxation. ${ }^{61}$ The thermal lens was probed using a $532 \mathrm{~nm}$ continuous wave laser (Coherent, Verdi V6), which was chosen to avoid spectral overlap with the wavelength tunable heating beam. A low probe power $(210 \mu \mathrm{W})$ was used to minimize steady-state heating of the nanostructure due to interband transitions at $532 \mathrm{~nm}$. Photothermal spectroscopy presents a general technique that can be applied to any strongly absorbing nanostructure to obtain its absorption spectrum without measuring scattering.
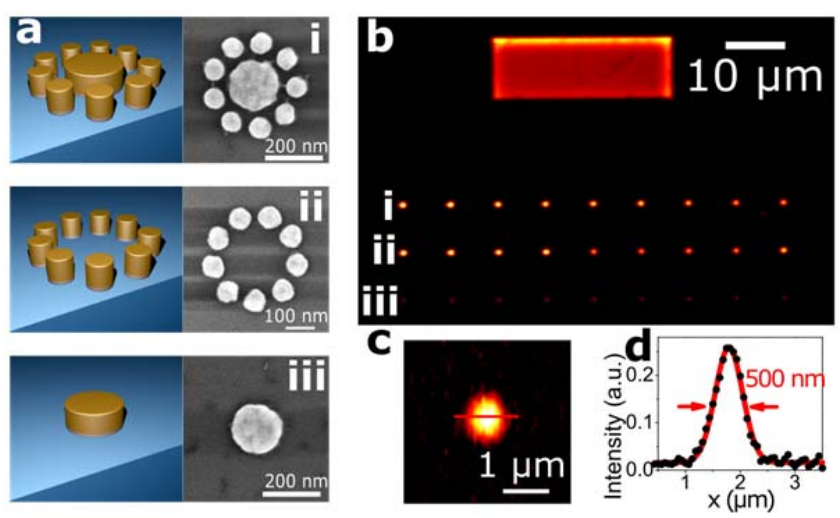

Figure 2. a) Schematic illustrations of the Fano cluster and its components (left) together with their corresponding SEM images (right). b) Photothermal image of a large sample area containing the lithographically prepared nanostructures as well as a gold bar used as a reference film. The roman numerals indicate the corresponding nanostructures in a. For the photothermal image, the wavelength of the heating laser was $700 \mathrm{~nm}$ (VIS channel) and the power was $\sim 71 \mu \mathrm{W}$. The probe laser wavelength and power were $532 \mathrm{~nm}$ and $210 \mu \mathrm{W}$, respectively. The resolution of the image was $210 \mathrm{~nm} /$ pixel. c) High resolution photothermal image of the central disk. The wavelength of the heating laser was $800 \mathrm{~nm}$ (NIR channel) and the power was $\sim 15 \mu \mathrm{W}$. The probe laser wavelength and power were $532 \mathrm{~nm}$ and $210 \mu \mathrm{W}$, respectively. The resolution of the image was $63 \mathrm{~nm} /$ pixel. d) Intensity crosssection of the photothermal image in c. The red line is a Gaussian fit yielding a FWHM of $500 \mathrm{~nm}$.

Fano decamers were fabricated on a glass substrate by electron-beam lithography. The decamer (Fig. 2a, i) was composed of a $175 \mathrm{~nm}$ diameter central disk (Fig. 2a, iii) surrounded by an outer ring of 9 smaller disks having equal diameters of $75 \mathrm{~nm}$ (Fig. 2a, ii). The thickness of 
gold was $35 \mathrm{~nm}$ and a $3 \mathrm{~nm}$ thick Ti layer was used for adhesion of gold to the glass substrate. The gap between each disk was designed to be $12 \mathrm{~nm}$, yielding an overall size of $\sim 350 \mathrm{~nm}$ for the decamer. Spacing individual decamers (i) and subunits, i.e. outer rings (ii) and central disks (iii), $8 \mu \mathrm{m}$ apart eliminated possible near-field and far-field interactions. The entire substrate was covered with glycerol, which is typically used in photothermal imaging to maximize the signal, ${ }^{61}$ by positioning the sample and substrate within a small liquid cell consisting of a second coverslip and a $50 \mu \mathrm{m}$ mylar spacer.

Prior to single-particle absorption spectroscopy, a raster-scanned photothermal image of the sample was obtained to identify the locations of the individual nanostructures (Fig. 2b). Each row in this image contained the same type of nanostructure as depicted by the roman numerals, corresponding to the decamer, outer ring, and the central disk, as shown in Fig. 2a. In addition to the equally spaced nanostructures that appear as spots in this image, the rectangular shape on the top corresponds to the photothermal signal of a $35 \mathrm{~nm}$ thick gold film. The continuous gold film was used to correct the measured absorption spectrum of the nanostructures, i.e. to account for wavelength-dependent signal variations that can arise from different laser powers and pumpprobe overlaps. With the simulated absorption spectrum of a gold film (Fig. S2) a straightforward correction factor was obtained, as described in detail in our previous work. ${ }^{60}$

To ensure homogeneous heating of each entire decamer structure during the absorption measurements, we employed an objective with a low numerical aperture of 0.7 to focus the heating and probe beams. The size of the overlapped beams was verified by taking a high resolution photothermal image of the central disk (Fig. 2c) and analyzing the intensity crosssection with a Gaussian function (Fig. 2d). We obtained a full width at half maximum (FWHM) along the horizontal and vertical directions of $\sim 600 \mathrm{~nm}$ and $\sim 500 \mathrm{~nm}$, respectively, which was 
significantly larger than the size of even the largest decamers, ensuring homogeneous excitation.

The slight asymmetry in the spot size was due to a non-perfect beam mode of the supercontinuum laser, which had no effect on the results shown here, as tested by rotating the sample by $90^{\circ}$.
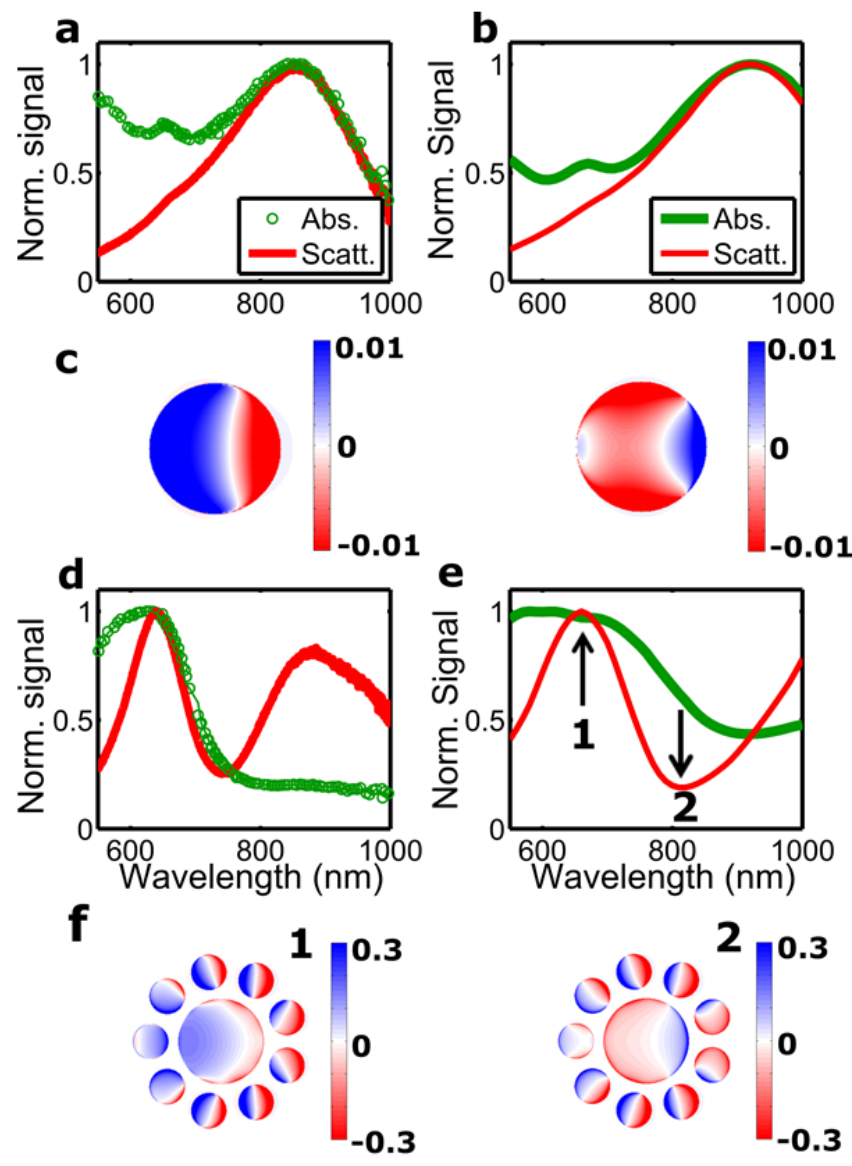

Figure 3. a) Comparison of the measured absorption (green circles) and measured scattering (red line) spectra for the same central disk. The absorption spectrum is cut-off at $550 \mathrm{~nm}$ to avoid interference with the $532 \mathrm{~nm}$ probe beam. b) Simulated absorption and scattering spectra of the central disk. c) Calculated charge distributions of the disk dipolar (left) and quadrupolar (right) plasmon mode. d) Comparison of measured absorption and scattering spectra for the same decamer. e) Simulated absorption and scattering spectra of the decamer. f) Charge distributions calculated at the spectral positions marked 1 and 2 in e, corresponding to the blue Fano peak at 660 and the Fano dip at $820 \mathrm{~nm}$, respectively. Heating beam powers varied between 5 and $150 \mu \mathrm{W}$ across the wavelength range and were adjusted through the use of temperature-balancing filters (Thorlabs, FGT165S), while the probe power at 532 nm was $210 \mu \mathrm{W}$. We checked that no photothermal damage occurred under these conditions by taking several scans and recording scattering spectra before and after photothermal spectroscopy. The spectra shown here correspond to unpolarized excitation by averaging two measurements with orthogonal linear excitation polarizations of the heating beam. ${ }^{60}$ Stitching the spectra from the VIS and NIR AOTF channels was accomplished as described in the SI. 
We first measured the wavelength-dependent photothermal response of an individual central disk in order to verify the operation of our wavelength extended single-particle absorption spectrometer. The absorption spectrum of the central disk shows a strong signal over a broad wavelength range and exhibits a dipole mode, and in addition a quadrupolar mode, which is absent from the corresponding scattering spectrum of the same individual disk (Fig. 3a). In the absorption spectrum of the central disk the dipolar mode is visible at $840 \mathrm{~nm}$ while the quadrupolar mode is seen at $650 \mathrm{~nm}$ with absorption increasing at shorter wavelengths due to interband transistions (Fig. 3a, green symbols). The scattering spectrum of the same central disk also shows the dipolar resonance at $840 \mathrm{~nm}$, spectrally overlapping with the absorption spectrum (Fig. 3a, red line). However, the quadrupolar mode is entirely absent from the scattering spectrum, apart from a very subtle shoulder that can be observed at $\sim 650 \mathrm{~nm}$. The reduced intensity of the quadrupolar mode in the scattering spectrum relative to the absorption spectrum arises from the subradiant nature of this plasmon mode. ${ }^{62}$ Interband transitions are also visible in the absorption spectrum but do not appear in the scattering spectrum, as would be expected. The single-particle scattering measurements were performed using a transmission dark-field microscope (see supporting information (SI)), and the same nanostructures could be found using alignment markers that were integrated into the sample design (Fig. S3).

The fact that we can observe the spectrum of a purely absorptive quadrupolar plasmon mode has potentially important implications. It has been reported that quadrupolar modes can be twice as sensitive to changes in the refractive index of their surrounding environment compared to dipolar modes. ${ }^{27,63}$ Quadrupole resonances monitored by this method may serve as improvedsensitivity nanoscale surface plasmon resonance sensors for detection of, for example, discrete binding events of individual proteins. ${ }^{64}$ 
Finite difference time domain (FDTD) simulations further validate the measured absorption spectrum of the central disk. We performed simulations using a commercial software package (Lumerical Solutions) based on the FDTD method (see SI for details). The experimentally observed dipolar and quadrupolar absorption as well as the onset of interband transitions at shorter wavelength are all well reproduced by the simulations (Fig. 3b, green line). The simulated scattering spectrum of the central disk also matches with the experimental result (Fig. 3b, red line). Simulated charge distributions (Fig. 3c) confirm the assignment of dipolar and quadrupolar plasmon modes. The overall lineshapes of the measured and simulated spectra are very similar with only minor resonance wavelength shifts and relative intensity differences. This small discrepancy is most likely due to the small structural inhomogeneities present in the lithographically fabricated gold nanodisks, due, for example, to polycrystallinity that cannot be resolved accurately within the resolution of scanning electron microscopy (SEM). Furthermore, the side walls of the lithographically prepared nanostructures typically possess a tilt angle, ${ }^{65}$ which can lead to a blueshift of the plasmon resonance compared to ideal nanostructures with perfectly sharp edges. The excellent agreement between all measured and simulated spectra for the same structure clearly validates our ability to measure absorption and scattering spectra over a broad wavelength range from 550 to $1000 \mathrm{~nm}$.

We now apply this approach to the more complex decamer Fano cluster. The physical mechanisms for formation of Fano resonances in coupled plasmonic systems have been extensively described. ${ }^{18}$ Briefly, in the language of molecular orbital theory, the sub- and superradiant modes are the adiabatic modes formed from hybridization of the diabatic dipolar modes of the ring and the larger central disk. Due to its nature as an interference of the sub- and superradiant modes, the position of the Fano dip does not coincide with the subradiant mode 
(except in the situation where the two modes are degenerate). The position of the Fano dip is located between these two modes thus cutting out a "hole" in the spectrum of the superradiant mode. Although the two maxima on either side of the Fano dip are associated with the same broad superradiant mode, they correspond to specific superpositions of the unhybridized diabatic ring and central disk modes. ${ }^{18}$ In the discussion below, we will refer to the low and high energy "peaks" as the red and blue Fano peaks respectively. The red Fano peak corresponds to the dipolar mode of the central disk and the blue Fano peak corresponds to the dipolar mode of the ring.

Compared to a simple disk, the absorption and scattering spectra of the decamer reveal even stronger differences in spectral lineshapes. The measured scattering spectrum of the decamer exhibits two Fano peaks at $\sim 650$ and $850 \mathrm{~nm}$ and a dip around $780 \mathrm{~nm}$, characteristic of a Fano resonance (Fig. 3d, red line). In contrast, the absorption spectrum of the decamer shows only one major peak at the subradiant mode around $660 \mathrm{~nm}$ (Fig. 3d, green symbols). This large difference in absorption and scattering lineshapes for the decamer is consistent with theoretical predictions but has not previously been observed experimentally.

FDTD simulations for the decamer similarly demonstrate distinct differences between absorption and scattering (Fig. 3d), in very good agreement with the experiments. The simulated scattering spectrum (Fig. 3e, red curve) displays a strong asymmetric lineshape with a Fano dip at $800 \mathrm{~nm}$, resulting from the interference of the superradiant and subradiant modes. This assignment was confirmed by the simulated charge distributions (Fig. 3f) at the blue Fano peak at $660 \mathrm{~nm}$ (arrow 1 in Fig. 3e) and at the Fano dip at $800 \mathrm{~nm}$ (arrow 2 in Fig. 3e). The charge distribution at $660 \mathrm{~nm}$ shows a parallel alignment of the dipole moments of all disks, i.e. the characteristics of superradiance. At $800 \mathrm{~nm}$, the dipole moments of the disk and the ring are out 
of phase, resulting in destructive interference and subradiance. ${ }^{21}$ The simulated absorption spectrum of the decamer (Fig. 3e, green line) also agrees well with the measured spectrum, exhibiting a single absorption feature around the subradiant mode. This absorption feature is made up of both the superradiant decamer mode and the quadrupolar modes of the central disk and therefore appears broad. A closer comparison between the simulated and measured spectra illustrates a similar blueshift as was observed for the central disk, and is most likely due to structural deviations from the idealized geometry used in the simulations.
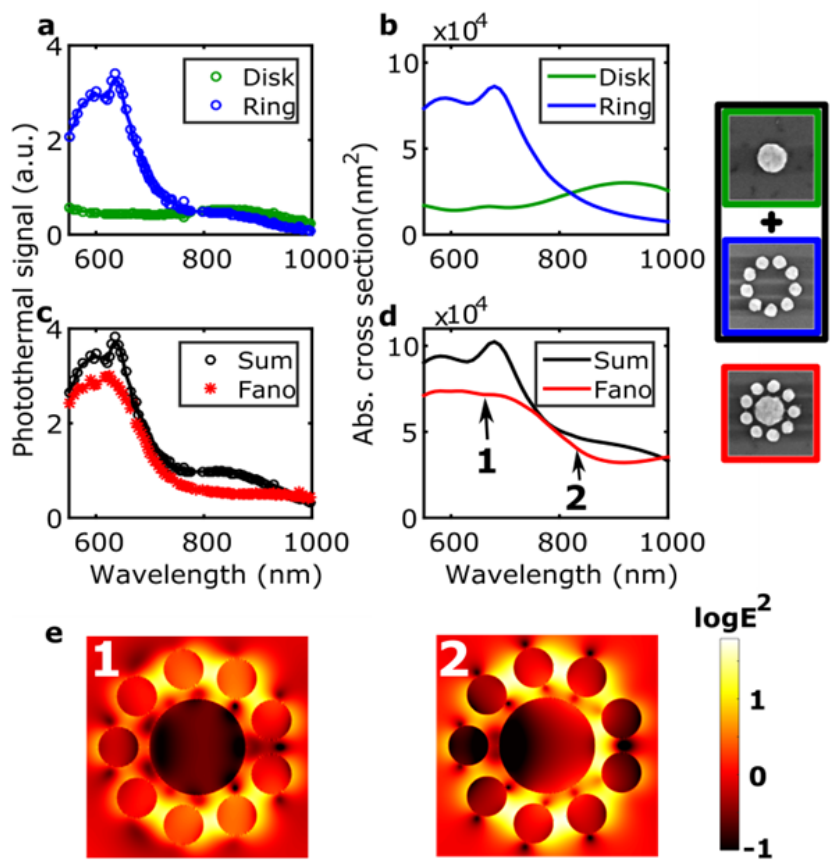

Figure 4. Decomposition of the Fano cluster absorption spectrum into its central disk and outer ring components. a) Measured absorption spectrum of the central disk (green) and the outer ring (blue). b) Calculated absorption spectrum of the central disk (green) and the outer ring (blue). c) Sum (black) of the measured central disk and outer ring absorption spectra compared to the absorption spectrum of the Fano cluster (red). d) Sum (black) of the calculated central disk outer ring absorption spectra compared to the calculated absorption spectrum of the Fano cluster (red). SEM images of the central disk, outer ring, and decamer with colored frames corresponding to the line colors of the spectra in a-d are shown for reference. The size of each SEM image is $500 \mathrm{~nm} \times 500 \mathrm{~nm}$. e) Electric field intensity maps of the Fano cluster calculated at positions 1 (left) and 2 (right) corresponding to the blue Fano peak and the Fano dip, respectively. Note that electric fields both inside and outside the gold nanodisks are given, and that fields outside are larger at 2 while fields inside are larger at 1 . Experimental parameters for the photothermal spectroscopy were the same as described for Fig. 3. 
Measuring the absorption spectra of the constituent components of the Fano cluster - the outer ring of nanodisks and the larger central disk - yields important insight into the non-radiative coupling of the involved plasmon modes (Fig. 4). The absorption spectrum of the outer ring exhibits two almost overlapping peaks in analogy with the bonding and anti-bonding modes of a homodimer. These two modes are clearly resolved in the measured (Fig. 4a) and simulated (Fig. 4b) absorption spectra. Fig. S4 further supports this assignment by showing simulated absorption and scattering spectra of the outer ring in comparison to a single disk and a homodimer. With the absorption spectrum of the central disk, included again in Figs. 4a and 4b, we can assess how the absorption spectrum of the Fano cluster differs from the sum of its components. Both experiment and simulations illustrate that the absorption spectrum changes when the outer ring and central disk are combined, based on near-field coupling between the nanodisks (Figs. 4c and 4d). However, unlike the result for scattering (Fig. S5), the difference is rather small and the absorption spectrum of the Fano cluster is dominated by the signal from the outer ring. The slight reduction in overall absorption in the interacting cluster compared to the sum of the absorption in its individual components is characteristic of any composite interacting plasmonic system. The absorption cross sections are larger than the physical cross sections so when two particles are close together, neither particle is able to absorb the same amount of light as if they were alone.

Hot electron generation by plasmon decay depends also on excitation properties. Strong plasmon absorption is therefore not the only factor that determines the yield of hot charge carriers. It has recently been shown that the yield of hot electrons is proportional to the square of the near-field inside the nanoparticles. ${ }^{59}$ We therefore calculated maps (Fig. 4e) of the near-field intensities both inside and outside the nanodisks for the Fano cluster at the blue Fano peak (position 1) and at the Fano dip (position 2). As predicted theoretically, ${ }^{66}$ the maximum near- 
field intensities outside the nanoparticles occur at the Fano dip (position 2), a property that has been extensively explored in surface enhanced spectroscopy and plasmon enhanced nonlinear

optics applications. ${ }^{21,42,43,67-70}$ Interestingly, the largest $\left|E_{\text {inside }}\right|^{2}$ is observed at the blue Fano peak (position 1) corresponding to the ring resonance where the absorption is also large. We therefore conclude that the highest yield of hot electrons for this decamer most likely occurs at the blue Fano peak because here absorption is maximized with strong internal electric fields.

\section{CONCLUSIONS}

Using our recently developed single-particle absorption spectroscopy, we were able to directly measure the absorption spectra of plasmonic Fano clusters over an extended wavelength range of $550-1000 \mathrm{~nm}$. We verified the theoretical predictions that while Fano interference may be present in the scattering spectrum of plasmonic nanostructures, it should be absent in the absorption spectrum. For a gold decamer consisting of a large central disk surrounded by a ring of smaller disks, the maximum absorption occurs on the blue side of the Fano resonance and occurs in the surrounding disks. The wavelength of maximum absorption coincides with the maximum near-field inside the gold nanodisks, and it is therefore the optimal wavelength for hot carrier generation. We anticipate that our results will promote further studies of the non-radiative properties of complex nanostructures to enable the structural optimization for light harvesting, sensing, and photocatalytic applications.

\section{ASSOCIATED CONTENT}

\section{Supporting information:}


Supporting Information Available: Experimental and simulation details, laser lines after the AOTFs, absorption spectrum of the gold film, sample design and mask, analysis of plasmon coupling in the outer ring, and decomposition of the Fano cluster scattering spectrum into its central disk and outer ring components. This material is available free of charge via the Internet at http://pubs.acs.org.

\section{AUTHOR INFORMATION}

\section{Corresponding Author}

*E-mail: slink@rice.edu

\section{Present Address}

${ }^{9}$ ASELSAN Research Center, Mehmet Akif Ersoy Mah. 296. Cad. No: 16, 06370 Yenimahalle, Ankara, Turkey

\section{Author Contributions}

The manuscript was written through contributions of all authors. All authors have given approval to the final version of the manuscript.

\section{Notes}

The authors declare no competing financial interest.

\section{ACKNOWLEDGMENT}

We acknowledge financial support from the Robert A. Welch Foundation (C-1220 to NJH, C1222 to PN, C-1664 to SL) the Army Research Office (MURI W911NF-12-1-0407), and the Air 
Force Office of Scientific Research (MURI FA9550-15-1-0022). M.Y.acknowledges financial support from the Smalley-Curl Institute at Rice University through a Carl\&Lillian Illig Postdoctoral Fellowship. A.H. acknowledges support from the National Science Foundation through a Graduate Research Fellowship (0940902). We thank Michael McClain for help with the artwork in Figure 1.

\footnotetext{
ABBREVIATIONS

AOTF, acousto optical tunable filter; FDTD, finite difference time domain; SEM, scanning electron microscope; FWHM, full width at half maximum
} 


\section{REFERENCES}

1. Willets, K. A.; Duyne, R. P. V.:Localized Surface Plasmon Resonance Spectroscopy and Sensing. Annu. Rev. Phys. Chem. 2007, 58, 267-297.

2. Mayer, K. M.; Hafner, J. H.:Localized Surface Plasmon Resonance Sensors. Chem. Rev. 2011, 111, 3828-3857.

3. $\quad$ Pryce, I. M.; Aydin, K.; Kelaita, Y. A.; Briggs, R. M.; Atwater, H. A.:Highly Strained Compliant Optical Metamaterials with Large Frequency Tunability. Nano Lett. 2010, 10, 42224227.

4. Yap, F. L.; Thoniyot, P.; Krishnan, S.; Krishnamoorthy, S.:Nanoparticle Cluster Arrays for High-Performance SERS through Directed Self-Assembly on Flat Substrates and on Optical Fibers. ACS Nano 2012, 6, 2056-2070.

5. Rycenga, M.; Camargo, P. H. C.; Li, W. Y.; Moran, C. H.; Xia, Y. N.:Understanding the SERS Effects of Single Silver Nanoparticles and Their Dimers, One at a Time. J. Phys. Chem. Lett. 2010, 1, 696-703.

6. Tian, C. F.; Ding, C. H.; Liu, S. Y.; Yang, S. C.; Song, X. P.; Ding, B. J.; Li, Z. Y.; Fang, J. X.:Nanoparticle Attachment on Silver Corrugated-Wire Nanoantenna for Large Increases of Surface-Enhanced Raman Scattering. ACS Nano 2011, 5, 9442-9449.

7. Kumar, K.; Duan, H.; Hegde, R. S.; Koh, S. C. W.; Wei, J. N.; Yang, J. K. W.:Printing colour at the optical diffraction limit. Nat Nano 2012, 7, 557-561.

8. Barrow, S. J.; Funston, A. M.; Gómez, D. E.; Davis, T. J.; Mulvaney, P.:Surface Plasmon Resonances in Strongly Coupled Gold Nanosphere Chains from Monomer to Hexamer. Nano Lett. 2011, 11, 4180-4187.

9. $\quad$ Byers, C. P.; Zhang, H.; Swearer, D. F.; Yorulmaz, M.; Hoener, B. S.; Huang, D.; Hoggard, A.; Chang, W.-S.; Mulvaney, P.; Ringe, E.; Halas, N. J.; Nordlander, P.; Link, S.; Landes, C. F.:From tunable core-shell nanoparticles to plasmonic drawbridges: Active control of nanoparticle optical properties. Science Advances 2015, 1, e1500988.

10. Herrmann, L. O.; Valev, V. K.; Tserkezis, C.; Barnard, J. S.; Kasera, S.; Scherman, O. A.; Aizpurua, J.; Baumberg, J. J.:Threading plasmonic nanoparticle strings with light. Nat Commun 2014, 5, 4568.

11. Slaughter, L. S.; Willingham, B. A.; Chang, W.-S.; Chester, M. H.; Ogden, N.; Link, S.:Toward Plasmonic Polymers. Nano Lett. 2012, 12, 3967-3972.

12. Fan, J. A.; Wu, C.; Bao, K.; Bao, J.; Bardhan, R.; Halas, N. J.; Manoharan, V. N.; Nordlander, P.; Shvets, G.; Capasso, F.:Self-Assembled Plasmonic Nanoparticle Clusters. Science 2010, 328, 1135-1138.

13. Lassiter, J. B.; Sobhani, H.; Fan, J. A.; Kundu, J.; Capasso, F.; Nordlander, P.; Halas, N. J.:Fano Resonances in Plasmonic Nanoclusters: Geometrical and Chemical Tunability. Nano Lett. 2010, 10, 3184-3189.

14. Miroshnichenko, A. E.; Flach, S.; Kivshar, Y. S.:Fano resonances in nanoscale structures. Rev. Mod. Phys. 2010, 82, 2257-2298.

15. Verellen, N.; Sonnefraud, Y.; Sobhani, H.; Hao, F.; Moshchalkov, V. V.; Dorpe, P. V.; Nordlander, P.; Maier, S. A.:Fano Resonances in Individual Coherent Plasmonic Nanocavities. Nano Lett. 2009, 9, 1663-1667.

16. Bakhti, S.; Destouches, N.; Tishchenko, A. V.:Coupled Mode Modeling To Interpret Hybrid Modes and Fano Resonances in Plasmonic Systems. ACS Photonics 2015, 2, 246-255. 
17. Funston, A. M.; Novo, C.; Davis, T. J.; Mulvaney, P.:Plasmon Coupling of Gold Nanorods at Short Distances and in Different Geometries. Nano Lett. 2009, 9, 1651-1658.

18. Lovera, A.; Gallinet, B.; Nordlander, P.; Martin, O. J. F.:Mechanisms of Fano Resonances in Coupled Plasmonic Systems. ACS Nano 2013, 7, 4527-4536.

19. Sonnichsen, C.; Reinhard, B. M.; Liphardt, J.; Alivisatos, A. P.:A molecular ruler based on plasmon coupling of single gold and silver nanoparticles. Nat Biotech 2005, 23, 741-745.

20. Lassiter, J. B.; Sobhani, H.; Knight, M. W.; Mielczarek, W. S.; Nordlander, P.; Halas, N. J.:Designing and Deconstructing the Fano Lineshape in Plasmonic Nanoclusters. Nano Lett. 2012, 12, 1058-1062.

21. Luk'yanchuk, B.; Zheludev, N. I.; Maier, S. A.; Halas, N. J.; Nordlander, P.; Giessen, H.; Chong, C. T.:The Fano resonance in plasmonic nanostructures and metamaterials. Nat Mater 2010, 9, 707-715.

22. Sonnefraud, Y.; Verellen, N.; Sobhani, H.; Vandenbosch, G. A. E.; Moshchalkov, V. V.; Van Dorpe, P.; Nordlander, P.; Maier, S. A.:Experimental Realization of Subradiant, Superradiant, and Fano Resonances in Ring/Disk Plasmonic Nanocavities. ACS Nano 2010, 4, 1664-1670.

23. Fano, U.:Effects of Configuration Interaction on Intensities and Phase Shifts. Phys. Rev. 1961, 124, 1866-1878.

24. Zhang, S.; Genov, D. a.; Wang, Y.; Liu, M.; Zhang, X.:Plasmon-Induced Transparency in Metamaterials. Phys. Rev. Lett. 2008, 101, 047401.

25. Liu, N.; Langguth, L.; Weiss, T.; Kästel, J.; Fleischhauer, M.; Pfau, T.; Giessen, H.:Plasmonic analogue of electromagnetically induced transparency at the Drude damping limit. Nat. Mater. 2009, 8, 758-762.

26. Artar, A.; Yanik, A. A.; Altug, H.:Multispectral Plasmon Induced Transparency in Coupled Meta-Atoms. Nano Lett. 2011, 11, 1685-1689.

27. Hao, F.; Nordlander, P.; Sonnefraud, Y.; Dorpe, P. V.; Maier, S. A.:Tunability of Subradiant Dipolar and Fano-Type Plasmon Resonances in Metallic Ring/Disk Cavities: Implications for Nanoscale Optical Sensing. ACS Nano 2009, 3, 643-652.

28. Huo, Y.; Jia, T.; Zhang, Y.; Zhao, H.; Zhang, S.; Feng, D.; Sun, Z.:Narrow and Deep Fano Resonances in a Rod and Concentric Square Ring-Disk Nanostructures. Sensors 2013, 13, $11350-11361$.

29. Bao, K.; Mirin, N. a.; Nordlander, P.:Fano resonances in planar silver nanosphere clusters. Appl. Phys. A 2010, 100, 333-339.

30. Hentschel, M.; Saliba, M.; Vogelgesang, R.; Giessen, H.; Alivisatos, a. P.; Liu, $\mathrm{N}$.:Transition from isolated to collective modes in plasmonic oligomers. Nano Lett. 2010, 10, 2721-2726.

31. Yan, B.; Boriskina, S. V.; Reinhard, B. M.:Optimizing Gold Nanoparticle Cluster Configurations $(\mathrm{n}<7)$ for Array Applications. J. Phys. Chem. C 2011, 115, 4578-4583.

32. Hentschel, M.; Dregely, D.; Vogelgesang, R.; Giessen, H.; Liu, N.:Plasmonic oligomers: the role of individual particles in collective behavior. ACS Nano 2011, 5, 2042-2050.

33. Dregely, D.; Hentschel, M.; Giessen, H.:Excitation and tuning of higher-order fano resonances in plasmonic oligomer clusters. ACS Nano 2011, 5, 8202-8211.

34. Rahmani, M.; Lukiyanchuk, B.; Ng, B.; Tavakkoli, A. K. G.; Liew, Y. F.; Hong, M. H.:Generation of Pronounced Fano Resonances and Tuning of Subwavelength Spatial Light Distribution in Plasmonic Pentamers. Opt. Express 2011, 19, 4949-4956. 
35. Rahmani, M.; Tahmasebi, T.; Lin, Y.; Lukiyanchuk, B.; Liew, T. Y. F.; Hong, M. H.:Influence of Plasmon Destructive Interferences on Optical Properties of Gold Planar Quadrumers. Nanotechnology 2011, 22, 245204.

36. Artar, A.; Yanik, A. A.; Altug, H.:Directional Double Fano Resonances in Plasmonic Hetero-Oligomers. Nano Lett. 2011, 11, 3694-3700.

37. Rahmani, M.; Lei, D. Y.; Giannini, V.; Lukiyanchuk, B.; Ranjbar, M.; Liew, T. Y. F.; Hong, M.; Maier, S. A.:Subgroup Decomposition of Plasmonic Resonances in Hybrid Oligomers: Modeling the Resonance Lineshape. Nano Lett. 2012, 12, 2101-2106.

38. Abb, M.; Wang, Y.; Albella, P.; de Groot, C. H.; Aizpurua, J.; Muskens, O.

L.:Interference, Coupling, and Nonlinear Control of High-Order Modes in Single Asymmetric Nanoantennas. ACS Nano 2012, 6, 6462-6470.

39. Mirin, N. A.; Bao, K.; Nordlander, P.:Fano Resonances in Plasmonic Nanoparticle Aggregates. J. Phys. Chem. A 2009, 113, 4028-4034.

40. Tamma, V. A.; Cui, Y.; Zhou, J.; Park, W.:Nanorod orientation dependence of tunable Fano resonance in plasmonic nanorod heptamers. Nanoscale 2013, 5, 1592-1602.

41. $\quad$ Chang, W.-S.; Lassiter, J. B.; Swanglap, P.; Sobhani, H.; Khatua, S.; Nordlander, P.; Halas, N. J.; Link, S.:A Plasmonic Fano Switch. Nano Lett. 2012, 12, 4977-4982.

42. Deng, H.-D.; Chen, X.-Y.; Xu, Y.; Miroshnichenko, A. E.:Single protein sensing with asymmetric plasmonic hexamer via Fano resonance enhanced two-photon luminescence. Nanoscale 2015, 7, 20405-20413.

43. He, J.; Fan, C.; Ding, P.; Zhu, S.; Liang, E.:Near-field engineering of Fano resonances in a plasmonic assembly for maximizing CARS enhancements. Sci. Rep. 2016, 6, 20777.

44. Khosravi Khorashad, L.; Besteiro, L. V.; Wang, Z.; Valentine, J.; Govorov, A. O.:Localization of Excess Temperature Using Plasmonic Hot Spots in Metal Nanostructures: Combining Nano-Optical Antennas with the Fano Effect. J. Phys. Chem. C 2016, 120, 1321513226.

45. Verellen, N.; Van Dorpe, P.; Huang, C.; Lodewijks, K.; Vandenbosch, G. A. E.; Lagae, L.; Moshchalkov, V. V.:Plasmon Line Shaping Using Nanocrosses for High Sensitivity Localized Surface Plasmon Resonance Sensing. Nano Lett. 2011, 11, 391-397.

46. Wu, C.; Khanikaev, A. B.; Adato, R.; Arju, N.; Yanik, A. A.; Altug, H.; Shvets, G.:Fanoresonant asymmetric metamaterials for ultrasensitive spectroscopy and identification of molecular monolayers. Nat Mater 2012, 11, 69-75.

47. Zhao, Y.; Hao, Q.; Ma, Y.; Lu, M.; Zhang, B.; Lapsley, M.; Khoo, I.-C.; Jun Huang, T.:Light-driven tunable dual-band plasmonic absorber using liquid-crystal-coated asymmetric nanodisk array. Appl. Phys. Lett. 2012, 100, 053119.

48. Fang, Z.; Liu, Z.; Wang, Y.; Ajayan, P. M.; Nordlander, P.; Halas, N. J.:GrapheneAntenna Sandwich Photodetector. Nano Lett. 2012, 12, 3808-3813.

49. Fang, Z.; Wang, Y.; Liu, Z.; Schlather, A.; Ajayan, P. M.; Koppens, F. H. L.; Nordlander, P.; Halas, N. J.:Plasmon-Induced Doping of Graphene. ACS Nano 2012, 6, 10222-10228.

50. Knight, M. W.; Sobhani, H.; Nordlander, P.; Halas, N. J.:Photodetection with Active Optical Antennas. Science 2011, 332, 702-704.

51. Fan, P.; Yu, Z.; Fan, S.; Brongersma, M. L.:Optical Fano resonance of an individual semiconductor nanostructure. Nat Mater 2014, 13, 471-475.

52. Linic, S.; Aslam, U.; Boerigter, C.; Morabito, M.:Photochemical transformations on plasmonic metal nanoparticles. Nat Mater 2015, 14, 567-576. 
53. Brongersma, M. L.; Halas, N. J.; Nordlander, P.:Plasmon-induced hot carrier science and technology. Nat Nano 2015, 10, 25-34.

54. Montgomery, J. M.; Lee, T.-W.; Gray, S. K.:Theory and modeling of light interactions with metallic nanostructures. J. Phys. 2008, 20, 323201.

55. Oubre, C.; Nordlander, P.:Optical properties of metallodielectric nanostructures calculated using the finite difference time domain method. J. Phys. Chem. B 2004, 108, 1774017747.

56. Gennaro, S. D.; Sonnefraud, Y.; Verellen, N.; Van Dorpe, P.; Moshchalkov, V. V.;

Maier, S. A.; Oulton, R. F.:Spectral interferometric microscopy reveals absorption by individual optical nanoantennas from extinction phase. Nat Commun 2014, 5, 3748.

57. Zhou, L.; Zhang, C.; McClain, M. J.; Manjavacas, A.; Krauter, C. M.; Tian, S.; Berg, F.; Everitt, H. O.; Carter, E. A.; Nordlander, P.; Halas, N. J.:Aluminum Nanocrystals as a Plasmonic Photocatalyst for Hydrogen Dissociation. Nano Lett. 2016, 16, 1478-1484.

58. Swearer, D. F.; Zhao, H.; Zhou, L.; Zhang, C.; Robatjazi, H.; Martirez, J. M. P.; Krauter, C. M.; Yazdi, S.; McClain, M. J.; Ringe, E.; Carter, E. A.; Nordlander, P.; Halas, N. J.:Heterometallic Antenna-Reactor Complexes for Photocatalysis. Proc. Natl. Acad. Sci. U.S.A. 2016, 113, 8916-8920.

59. Zheng, B. Y.; Zhao, H.; Manjavacas, A.; McClain, M.; Nordlander, P.; Halas, N.

J.:Distinguishing between plasmon-induced and photoexcited carriers in a device geometry. Nat Commun 2015, 6, 7797.

60. Yorulmaz, M.; Nizzero, S.; Hoggard, A.; Wang, L.-Y.; Cai, Y.-Y.; Su, M.-N.; Chang, W.-S.; Link, S.:Single-Particle Absorption Spectroscopy by Photothermal Contrast. Nano Lett. 2015, 15, 3041-3047.

61. Gaiduk, A.; Ruijgrok, P. V.; Yorulmaz, M.; Orrit, M.:Detection limits in photothermal microscopy. Chem. Sci. 2010, 1, 343-350.

62. Hägglund, C.; Zäch, M.; Petersson, G.; Kasemo, B.:Electromagnetic coupling of light into a silicon solar cell by nanodisk plasmons. Appl. Phys. Lett. 2008, 92, 053110.

63. Bastús, N. G.; Piella, J.; Puntes, V.:Quantifying the Sensitivity of Multipolar (Dipolar, Quadrupolar, and Octapolar) Surface Plasmon Resonances in Silver Nanoparticles: The Effect of Size, Composition, and Surface Coating. Langmuir 2016, 32, 290-300.

64. Zijlstra, P.; Paulo, P. M. R.; Orrit, M.:Optical detection of single non-absorbing molecules using the surface plasmon resonance of a gold nanorod. Nat Nano 2012, 7, 379-382. 65. Chang, W.-S.; Wen, F.; Chakraborty, D.; Su, M.-N.; Zhang, Y.; Shuang, B.; Nordlander, P.; Sader, J. E.; Halas, N. J.; Link, S.:Tuning the acoustic frequency of a gold nanodisk through its adhesion layer. Nat Commun 2015, 6, 7022.

66. Gallinet, B.; Siegfried, T.; Sigg, H.; Nordlander, P.; Martin, O. J. F.:Plasmonic Radiance: Probing Structure at the Ångström Scale with Visible Light. Nano Lett. 2013, 13, 497-503.

67. Zhang, Y.; Zhen, Y.-R.; Neumann, O.; Day, J. K.; Nordlander, P.; Halas, N. J.:Coherent anti-Stokes Raman scattering with single-molecule sensitivity using a plasmonic Fano resonance. Nat Commun 2014, 5, 4424.

68. Ye, J.; Wen, F.; Sobhani, H.; Lassiter, J. B.; Dorpe, P. V.; Nordlander, P.; Halas, N. J.:Plasmonic Nanoclusters: Near Field Properties of the Fano Resonance Interrogated with SERS. Nano Lett. 2012, 12, 1660-1667.

69. Thyagarajan, K.; Butet, J.; Martin, O. J. F.:Augmenting Second Harmonic Generation Using Fano Resonances in Plasmonic Systems. Nano Lett. 2013, 13, 1847-1851. 
70. Liu, S.-D.; Leong, E. S. P.; Li, G.-C.; Hou, Y.; Deng, J.; Teng, J. H.; Ong, H. C.; Lei, D. Y.:Polarization-Independent Multiple Fano Resonances in Plasmonic Nonamers for MultimodeMatching Enhanced Multiband Second-Harmonic Generation. ACS Nano 2016, 10, 1442-1453. 
TOC Figure

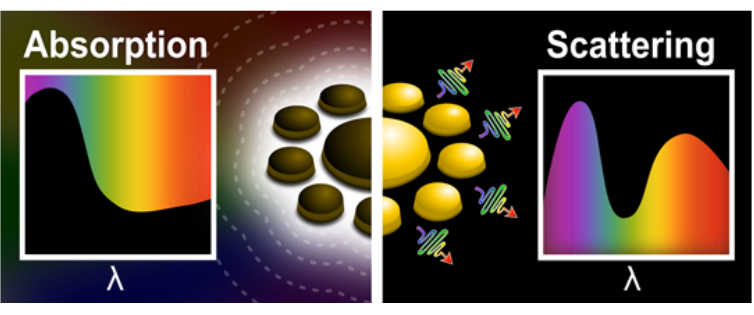

